\title{
An Iterative Algorithm Combining Viscosity Method with Parallel Method for a Generalized Equilibrium Problem and Strict Pseudocontractions
}

\author{
Jian-Wen Peng, ${ }^{1}$ Yeong-Cheng Liou, ${ }^{2}$ and Jen-Chih Yao $^{3}$ \\ ${ }^{1}$ College of Mathematics and Computer Science, Chongqing Normal University, Chongqing 400047, China \\ ${ }^{2}$ Department of Information Management, Cheng Shiu University, Kaohsiung, Taiwan 833, Taiwan \\ ${ }^{3}$ Department of Applied Mathematics, National Sun Yat-sen University, Kaohsiung, Taiwan 804, Taiwan
}

Correspondence should be addressed to Yeong-Cheng Liou, simplex_liou@hotmail.com

Received 5 August 2008; Accepted 4 January 2009

Recommended by Hichem Ben-El-Mechaiekh

We introduce a new approximation scheme combining the viscosity method with parallel method for finding a common element of the set of solutions of a generalized equilibrium problem and the set of fixed points of a family of finitely strict pseudocontractions. We obtain a strong convergence theorem for the sequences generated by these processes in Hilbert spaces. Based on this result, we also get some new and interesting results. The results in this paper extend and improve some well-known results in the literature.

Copyright (c) 2009 Jian-Wen Peng et al. This is an open access article distributed under the Creative Commons Attribution License, which permits unrestricted use, distribution, and reproduction in any medium, provided the original work is properly cited.

\section{Introduction}

Let $H$ be a real Hilbert space with inner product $\langle\cdot, \cdot\rangle$ and induced norm $\|\cdot\|$, and let $C$ be a nonempty-closed convex subset of $H$. Let $\varphi: H \rightarrow R \cup\{+\infty\}$ be a function and let $F$ be a bifunction from $C \times C$ to $R$ such that $C \cap \operatorname{dom} \varphi \neq \varnothing$, where $R$ is the set of real numbers and $\operatorname{dom} \varphi=\{x \in H: \varphi(x)<+\infty\}$. Flores-Bazán [1] introduced the following generalized equilibrium problem:

$$
\text { Find } x \in C \text { such that } F(x, y)+\varphi(y) \geq \varphi(x), \quad \forall y \in C \text {. }
$$

The set of solutions of $(1.1)$ is denoted by $\operatorname{GEP}(F, \varphi)$. Flores-Bazán [1] provided some characterizations of the nonemptiness of the solution set for problem (1.1) in reflexive Banach spaces in the quasiconvex case. Bigi et al. [2] studied a dual problem associated with the problem (1.1) with $C=H=R^{n}$. 
Let $\varphi(x)=\delta_{C}(x), \forall x \in H$. Here $\delta_{C}$ denotes the indicator function of the set $C$; that is, $\delta_{C}(x)=0$ if $x \in C$ and $\delta_{C}(x)=+\infty$ otherwise. Then the problem (1.1) becomes the following equilibrium problem:

$$
\text { Finding } x \in C \text { such that } F(x, y) \geq 0, \quad \forall y \in C \text {. }
$$

The set of solutions of (1.2) is denoted by $\mathrm{EP}(F)$. The problem (1.2) includes, as special cases, the optimization problem, the variational inequality problem, the fixed point problem, the nonlinear complementarity problem, the Nash equilibrium problem in noncooperative games, and the vector optimization problem. For more detail, please see [3-5] and the references therein.

If $F(x, y)=g(y)-g(x)$ for all $x, y \in C$, where $g: C \rightarrow R$ is a function, then the problem (1.1) becomes a problem of finding $x \in C$ which is a solution of the following minimization problem:

$$
\min _{y \in C}\{\varphi(y)+g(y)\}
$$

The set of solutions of (1.3) is denoted by $\operatorname{Argmin}(g, \varphi)$.

If $\varphi: H \rightarrow R \cup\{+\infty\}$ is replaced by a real-valued function $\phi: C \rightarrow R$, the problem (1.1) reduces to the following mixed equilibrium problem introduced by Ceng and Yao [6]:

$$
\text { Find } x \in C \text { such that } F(x, y)+\phi(y)-\phi(x) \geq 0, \quad \forall y \in C \text {. }
$$

Recall that a mapping $T: C \rightarrow C$ is said to be a $\kappa$-strict pseudocontraction [7] if there exists $0 \leq \kappa<1$, such that

$$
\|T x-T y\|^{2} \leq\|x-y\|^{2}+\kappa\|(I-T) x-(I-T) y\|^{2}, \quad \forall x, y \in C,
$$

where $I$ denotes the identity operator on $C$. When $\kappa=0, T$ is said to be nonexpansive. Note that the class of strict pseudocontraction mappings strictly includes the class of nonexpansive mappings. We denote the set of fixed points of $S$ by $\operatorname{Fix}(S)$.

Ceng and Yao [6], Yao et al. [8], and Peng and Yao $[9,10]$ introduced some iterative schemes for finding a common element of the set of solutions of the mixed equilibrium problem (1.4) and the set of common fixed points of a family of finitely (infinitely) nonexpansive mappings (strict pseudocontractions) in a Hilbert space and obtained some strong convergence theorems(weak convergence theorems). Some methods have been proposed to solve the problem (1.2); see, for instance, [3-5, 11-18] and the references therein. Recently, S. Takahashi and W. Takahashi [12] introduced an iterative scheme by the viscosity approximation method for finding a common element of the set of solutions of problem (1.2) and the set of fixed points of a nonexpansive mapping in a Hilbert space and proved a strong convergence theorem. Su et al. [13] introduced an iterative scheme by the viscosity approximation method for finding a common element of the set of solutions of problem (1.2) and the set of fixed points of a nonexpansive mapping and the set of solutions of the variational inequality problem for an $\alpha$-inverse strongly monotone mapping in a Hilbert space. Tada and Takahashi [14] introduced two iterative schemes for finding 
a common element of the set of solutions of problem (1.2) and the set of fixed points of a nonexpansive mapping in a Hilbert space and obtained both strong convergence theorem and weak convergence theorem. Ceng et al. [15] introduced an iterative algorithm for finding a common element of the set of solutions of problem (1.2) and the set of fixed points of a strict pseudocontraction mapping. Chang et al. [16] introduced some iterative processes based on the extragradient method for finding the common element of the set of fixed points of a family of infinitely nonexpansive mappings, the set of problem (1.2), and the set of solutions of a variational inequality problem for an $\alpha$-inverse strongly monotone mapping. Colao et al. [17] introduced an iterative method for finding a common element of the set of solutions of problem (1.2) and the set of fixed points of a finite family of nonexpansive mappings in a Hilbert space and proved the strong convergence of the proposed iterative algorithm to the unique solution of a variational inequality, which is the optimality condition for a minimization problem. To the best of our knowledge, there is not any algorithms for solving problem (1.1).

On the other hand, Marino and $\mathrm{Xu}$ [19] and Zhou [20] introduced and researched some iterative scheme for finding a fixed point of a strict pseudocontraction mapping. Acedo and $\mathrm{Xu}$ [21] introduced some parallel and cyclic algorithms for finding a common fixed point of a family of finite strict pseudocontraction mappings and obtained both weak and strong convergence theorems for the sequences generated by the iterative schemes.

In the present paper, we introduce a new approximation scheme combining the viscosity method with parallel method for finding a common element of the set of solutions of the generalized equilibrium problem and the set of fixed points of a family of finitely strict pseudocontractions. We obtain a strong convergence theorem for the sequences generated by these processes. Based on this result, we also get some new and interesting results. The results in this paper extend and improve some well-known results in the literature.

\section{Preliminaries}

Let $H$ be a real Hilbert space with inner product $\langle\cdot, \cdot\rangle$ and norm $\|\cdot\|$. Let $C$ be a nonemptyclosed convex subset of $H$. Let symbols $\rightarrow$ and $\rightarrow$ denote strong and weak convergences, respectively. In a real Hilbert space $H$, it is well known that

$$
\|\lambda x+(1-\lambda) y\|^{2}=\lambda\|x\|^{2}+(1-\lambda)\|y\|^{2}-\lambda(1-\lambda)\|x-y\|^{2}
$$

for all $x, y \in H$ and $\lambda \in[0,1]$.

For any $x \in H$, there exists a unique nearest point in $C$, denoted by $P_{C}(x)$, such that $\left\|x-P_{C}(x)\right\| \leq\|x-y\|$ for all $y \in C$. The mapping $P_{C}$ is called the metric projection of $H$ onto $C$. We know that $P_{C}$ is a nonexpansive mapping from $H$ onto $C$. It is also known that $P_{C} x \in C$ and

$$
\left\langle x-P_{C}(x), P_{C}(x)-y\right\rangle \geq 0
$$

for all $x \in H$ and $y \in C$.

For each $B \subseteq H$, we denote by $\operatorname{conv}(B)$ the convex hull of $B$. A multivalued mapping $G: B \rightarrow 2^{H}$ is said to be a KKM map if, for every finite subset $\left\{x_{1}, x_{2}, \ldots, x_{n}\right\} \subseteq B$, $\operatorname{conv}\left(\left\{x_{1}, x_{2}, \ldots, x_{n}\right\}\right) \subseteq \bigcup_{n=1}^{\infty} G\left(x_{i}\right)$. 
We will use the following results in the sequel.

Lemma 2.1 (see [22]). Let $B$ be a nonempty subset of a Hausdorff topological vector space $X$ and let $G: B \rightarrow 2^{X}$ be a KKM map. If $G(x)$ is closed for all $x \in B$ and is compact for at least one $x \in B$, then $\bigcap_{x \in B} G(x) \neq \varnothing$.

For solving the generalized equilibrium problem, let us give the following assumptions for the bifunction $F, \varphi$, and the set $C$ :

(A1) $F(x, x)=0$ for all $x \in C$;

(A2) $F$ is monotone, that is, $F(x, y)+F(y, x) \leq 0$ for any $x, y \in C$;

(A3) for each $y \in C, x \mapsto F(x, y)$ is weakly upper semicontinuous;

(A4) for each $x \in C, y \mapsto F(x, y)$ is convex;

(A5) for each $x \in C, y \mapsto F(x, y)$ is lower semicontinuous;

(B1) For each $x \in H$ and $r>0$, there exist a bounded subset $D_{x} \subseteq C$ and $y_{x} \in C \cap \operatorname{dom} \varphi$ such that for any $z \in C \backslash D_{x}$,

$$
F\left(z, y_{x}\right)+\varphi\left(y_{x}\right)+\frac{1}{r}\left\langle y_{x}-z, z-x\right\rangle<\varphi(z)
$$

(B2) $C$ is a bounded set.

Lemma 2.2. Let $C$ be a nonempty-closed convex subset of $H$. Let $F$ be a bifunction from $C \times C$ to $R$ satisfying (A1)-(A4) and let $\varphi: H \rightarrow R \cup\{+\infty\}$ be a proper lower semicontinuous and convex function such that $C \cap \operatorname{dom} \varphi \neq \varnothing$. For $r>0$ and $x \in H$, define a mapping $S_{r}: H \rightarrow C$ as follows:

$$
S_{r}(x)=\left\{z \in C: F(z, y)+\varphi(y)+\frac{1}{r}\langle y-z, z-x\rangle \geq \varphi(z), \forall y \in C\right\}
$$

for all $x \in H$. Assume that either (B1) or (B2) holds. Then, the following conclusions hold:

(1) for each $x \in H, S_{r}(x) \neq \varnothing$;

(2) $S_{r}$ is single-valued;

(3) $S_{r}$ is firmly nonexpansive, that is, for any $x, y \in H$,

$$
\left\|S_{r}(x)-S_{r}(y)\right\|^{2} \leq\left\langle S_{r}(x)-S_{r}(y), x-y\right\rangle ;
$$

(4) $\operatorname{Fix}\left(S_{r}\right)=\operatorname{GEP}(F, \varphi)$;

(5) $\operatorname{GEP}(F, \varphi)$ is closed and convex.

Proof. Let $x_{0}$ be any given point in $E$. For each $y \in C$, we define

$$
G(y)=\left\{z \in C: F(z, y)+\varphi(y)+\frac{1}{r}\left\langle y-z, z-x_{0}\right\rangle \geq \varphi(z)\right\} .
$$


Note that for each $y \in C$ ndom $\varphi, G(y)$ is nonempty since $y \in G(y)$ and for each $y \in C \backslash \operatorname{dom} \varphi$, $G(y)=C$. We will prove that $G$ is a KKM map on $C \cap \operatorname{dom} \varphi$. Suppose that there exists a finite subset $\left\{y_{1}, y_{2}, \ldots, y_{n}\right\}$ of $C \cap \operatorname{dom} \varphi$ and $\mu_{i} \geq 0$ for all $i=1,2, \ldots, n$ with $\sum_{i=1}^{n} \mu_{i}=1$ such that $\widehat{z}=\sum_{i=1}^{n} \mu_{i} y_{i} \notin G\left(y_{i}\right)$ for each $i=1,2, \ldots, n$. Then we have

$$
F\left(\widehat{z}, y_{i}\right)+\varphi\left(y_{i}\right)-\varphi(\widehat{z})+\frac{1}{r}\left\langle y_{i}-\widehat{z}, \widehat{z}-x_{0}\right\rangle<0
$$

for each $i=1,2, \ldots, n$. By (A4) and the convexity of $\varphi$, we have

$$
\begin{aligned}
0 & =F(\widehat{z}, \widehat{z})+\varphi(\widehat{z})-\varphi(\widehat{z})+\frac{1}{r}\left\langle\widehat{z}-\widehat{z}, \widehat{z}-x_{0}\right\rangle \\
& \leq \sum_{i=1}^{n} \mu_{i}\left[F\left(\widehat{z}, y_{i}\right)+\varphi\left(y_{i}\right)-\varphi(\widehat{z})\right]+\frac{1}{r}\left[\sum_{i=1}^{n} \mu_{i}\left\langle y_{i}-\widehat{z}, \widehat{z}-x_{0}\right\rangle\right]<0,
\end{aligned}
$$

which is a contradiction. Hence, $G$ is a KKM map on $C \cap \operatorname{dom} \varphi$. Note that $\overline{G(y)}^{w}$ (the weak closure of $G(y))$ is a weakly closed subset of $C$ for each $y \in C$. Moreover, if (B2) holds, then $\overline{G(y)}^{w}$ is also weakly compact for each $y \in C$. If (B1) holds, then for $x_{0} \in E$, there exists a bounded subset $D_{x_{0}} \subseteq C$ and $y_{x_{0}} \in C \cap \operatorname{dom} \varphi$ such that for any $z \in C \backslash D_{x_{0}}$,

$$
F\left(z, y_{x_{0}}\right)+\varphi\left(y_{x_{0}}\right)+\frac{1}{r}\left\langle y_{x_{0}}-z, z-x_{0}\right\rangle<\varphi(z)
$$

This shows that

$$
G\left(y_{x_{0}}\right)=\left\{z \in C: F\left(z, y_{x_{0}}\right)+\varphi\left(y_{x_{0}}\right)+\frac{1}{r}\left\langle y_{x_{0}}-z, z-x_{0}\right\rangle \geq \varphi(z)\right\} \subseteq D_{x_{0}}
$$

Hence, $\overline{G\left(y_{x_{0}}\right)}$ is weakly compact. Thus, in both cases, we can use Lemma 2.1 and have $\bigcap_{y \in \operatorname{Cndom} \varphi} \overline{G(y)} \neq \varnothing$.

Next, we will prove that $\overline{G(y)}^{w}=G(y)$ for each $y \in C$; that is, $G(y)$ is weakly closed. Let $z \in \overline{G(y)}^{w}$ and let $z_{m}$ be a sequence in $G(y)$ such that $z_{m} \rightarrow z$. Then,

$$
F\left(z_{m}, y\right)+\varphi(y)+\frac{1}{r}\left\langle y-z_{m}, z_{m}-x_{0}\right\rangle \geq \varphi\left(z_{m}\right) .
$$

Since $\|\cdot\|^{2}$ is weakly lower semicontinuous, we can show that

$$
\limsup _{m \rightarrow \infty}\left\langle y-z_{m}, z_{m}-x_{0}\right\rangle \leq\left\langle z-y, x_{0}-z\right\rangle
$$


It follows from (A3) and the weak lower semicontinuity of $\varphi$ that

$$
\begin{aligned}
\varphi(z) & \leq \liminf _{m \rightarrow \infty} \varphi\left(z_{m}\right) \leq \limsup _{m \rightarrow \infty}\left[F\left(z_{m}, y\right)+\varphi(y)+\frac{1}{r}\left\langle y-z_{m}, z_{m}-x_{0}\right\rangle\right] \\
& \leq \limsup _{m \rightarrow \infty}\left[F\left(z_{m}, y\right)+\varphi(y)\right]+\frac{1}{r} \limsup _{m \rightarrow \infty}\left\langle y-z_{m}, z_{m}-x_{0}\right\rangle \\
& \leq F(z, y)+\varphi(y)+\frac{1}{r}\left\langle z-y, x_{0}-z\right\rangle .
\end{aligned}
$$

This implies that $z \in G(y)$. Hence, $G(y)$ is weakly closed. Hence, $S_{r}\left(x_{0}\right)=\bigcap_{y \in C} G(y)=$ $\bigcap_{y \in \operatorname{Cndom} \varphi} G(y)=\bigcap_{y \in \operatorname{Cndom} \varphi} \overline{G(y)}^{w} \neq \varnothing$. Hence, from the arbitrariness of $x_{0}$, we conclude that $S_{r}(x) \neq \varnothing, \forall x \in H$.

We observe that $S_{r}(x) \subseteq \operatorname{dom} \varphi$. So by similar argument with that in the proof of Lemma 2.3 in [9], we can easily show that $S_{r}$ is single-valued and $S_{r}$ is a firmly nonexpansivetype map. Next, we claim that $\operatorname{Fix}\left(S_{r}\right)=\operatorname{GEP}(F, \varphi)$. Indeed, we have the following:

$$
\begin{aligned}
u \in \operatorname{Fix}\left(S_{r}\right) & \Longleftrightarrow u=S_{r}(u) \\
& \Longleftrightarrow F(u, y)+\varphi(y)+\frac{1}{r}\langle y-u, u-u\rangle \geq \varphi(u), \quad \forall y \in C \\
& \Longleftrightarrow F(u, y)+\varphi(y) \geq \varphi(u), \quad \forall y \in C \\
& \Longleftrightarrow u \in \operatorname{GEP}(F, \varphi) .
\end{aligned}
$$

At last, we claim that $\operatorname{GEP}(F, \varphi)$ is a closed convex. Indeed, Since $S_{r}$ is firmly nonexpansive, $S_{r}$ is also nonexpansive. By [23, Proposition 5.3], we know that $\operatorname{GEP}(F, \varphi)=\operatorname{Fix}\left(S_{r}\right)$ is closed and convex.

Remark 2.3. It is easy to see that Lemma 2.2 is a generalization of [9, Lemma 2.3].

Lemma 2.4 (see $[24,25])$. Assume that $\left\{\alpha_{n}\right\}$ is a sequence of nonnegative real numbers such that

$$
\alpha_{n+1} \leq\left(1-\gamma_{n}\right) \alpha_{n}+\delta_{n}
$$

where $\gamma_{n}$ is a sequence in $(0,1)$ and $\left\{\delta_{n}\right\}$ is a sequence such that

$$
\begin{aligned}
& \text { (i) } \sum_{n=1}^{\infty} \gamma_{n}=\infty ; \\
& \text { (ii) } \limsup _{n \rightarrow \infty} \frac{\delta_{n}}{\gamma_{n}} \leq 0 \text { or } \sum_{n=1}^{\infty}\left|\delta_{n}\right|<\infty .
\end{aligned}
$$

Then, $\lim _{n \rightarrow \infty} \alpha_{n}=0$.

Lemma 2.5. In a real Hilbert space $H$, there holds the following inequality:

$$
\|x+y\|^{2} \leq\|x\|^{2}+2\langle y, x+y\rangle
$$

for all $x, y \in H$. 


\section{Strong Convergence Theorems}

In this section, we show a strong convergence of an iterative algorithm based on both viscosity approximation method and parallel method which solves the problem of finding a common element of the set of solutions of a generalized equilibrium problem and the set of fixed points of a family of finitely strict pseudocontractions in a Hilbert space.

We need the following assumptions for the parameters $\left\{\gamma_{n}\right\},\left\{r_{n}\right\},\left\{\alpha_{n}\right\},\left\{\zeta_{1}^{(n)}\right\}$, $\left\{\zeta_{2}^{(n)}\right\}, \ldots,\left\{\zeta_{N}^{(n)}\right\}$, and $\left\{\beta_{n}\right\}$ :

(C1) $\lim _{n \rightarrow \infty} \alpha_{n}=0$ and $\sum_{n=1}^{\infty} \alpha_{n}=\infty$;

(C2) $1>\lim \sup _{n \rightarrow \infty} \beta_{n} \geq \liminf _{n \rightarrow \infty} \beta_{n}>0$;

(C3) $\left\{\gamma_{n}\right\} \subset[c, d]$ for some $c, d \in(\varepsilon, 1)$ and $\lim _{n \rightarrow \infty}\left|\gamma_{n+1}-\gamma_{n}\right|=0$;

(C4) $\liminf _{n \rightarrow \infty} r_{n}>0$ and $\lim _{n \rightarrow \infty}\left|r_{n+1}-r_{n}\right|=0$;

(C5) $\lim _{n \rightarrow \infty}\left|\zeta_{j}^{(n+1)}-\zeta_{j}^{(n)}\right|=0$ for all $j=1,2, \ldots, N$.

Theorem 3.1. Let $C$ be a nonempty-closed convex subset of a real Hilbert space $H$. Let $F$ be a bifunction from $C \times C$ to $R$ satisfying (A1)-(A5), and let $\varphi: C \rightarrow R \cup\{+\infty\}$ be a proper lower semicontinuous and convex function such that $C \cap \operatorname{dom} \varphi \neq \varnothing$. Let $N \geq 1$ be an integer. For each $1 \leq j \leq N$, let $T_{j}: C \rightarrow C$ be an $\varepsilon_{j}$-strict pseudocontraction for some $0 \leq \varepsilon_{j}<1$ such that $\Omega=\bigcap_{j=1}^{N} \operatorname{Fix}\left(T_{j}\right) \cap \operatorname{GEP}(F, \varphi) \neq \varnothing$. Assume for each $n,\left\{\zeta_{j}^{(n)}\right\}_{j=1}^{N}$ is a finite sequence of positive numbers such that $\sum_{j=1}^{N} \zeta_{j}^{(n)}=1$ for all $n$ and $\inf _{n \geq 1} \zeta_{j}^{(n)}>0$ for all $0 \leq j \leq N$. Let $\varepsilon=\max \left\{\varepsilon_{j}: 1 \leq j \leq N\right\}$. Assume that either (B1) or (B2) holds. Let $f$ be a contraction of $C$ into itself and let $\left\{x_{n}\right\},\left\{u_{n}\right\}$, and $\left\{y_{n}\right\}$ be sequences generated by

$$
\begin{gathered}
x_{1}=x \in C \\
F\left(u_{n}, y\right)+\varphi(y)+\frac{1}{r_{n}}\left\langle y-u_{n}, u_{n}-x_{n}\right\rangle \geq \varphi\left(u_{n}\right), \quad \forall y \in C, \\
y_{n}=\gamma_{n} u_{n}+\left(1-\gamma_{n}\right) \sum_{j=1}^{N} \zeta_{j}^{(n)} T_{j} u_{n} \\
x_{n+1}=\alpha_{n} f\left(x_{n}\right)+\beta_{n} x_{n}+\left(1-\alpha_{n}-\beta_{n}\right) y_{n}
\end{gathered}
$$

for every $n=1,2, \ldots$, where $\left\{\gamma_{n}\right\},\left\{r_{n}\right\},\left\{\alpha_{n}\right\},\left\{\zeta_{1}^{(n)}\right\},\left\{\zeta_{2}^{(n)}\right\}, \ldots,\left\{\zeta_{N}^{(n)}\right\}$, and $\left\{\beta_{n}\right\}$ are sequences of numbers satisfying the conditions (C1)-(C5). Then, $\left\{x_{n}\right\},\left\{u_{n}\right\}$, and $\left\{y_{n}\right\}$ converge strongly to $w=$ $P_{\Omega} f(w)$.

Proof. We show that $P_{\Omega} f$ is a contraction of $C$ into itself. In fact, there exists $a \in[0,1)$ such that $\|f(x)-f(y)\| \leq a\|x-y\|$ for all $x, y \in C$. So, we have

$$
\left\|P_{\Omega} f(x)-P_{\Omega} f(y)\right\| \leq\|f(x)-f(y)\| \leq a\|x-y\|
$$

for all $x, y \in C$. Since $H$ is complete, there exists a unique element $u_{0} \in C$ such that $u_{0}=$ $P_{\Omega} f\left(u_{0}\right)$. 
Let $u \in \Omega$ and let $\left\{S_{r_{n}}\right\}$ be a sequence of mappings defined as in Lemma 2.2. From $u_{n}=S_{r_{n}}\left(x_{n}\right) \in C$, we have

$$
\left\|u_{n}-u\right\|=\left\|S_{r_{n}}\left(x_{n}\right)-S_{r_{n}}(u)\right\| \leq\left\|x_{n}-u\right\|
$$

We define a mapping $W_{n}$ by

$$
W_{n} x=\sum_{j=1}^{N} \zeta_{j}^{(n)} T_{j} x, \quad \forall x \in C .
$$

By [21, Proposition 2.6], we know that $W_{n}$ is an $\varepsilon$-strict pseudocontraction and $F\left(W_{n}\right)=$ $\bigcap_{j=1}^{N} \operatorname{Fix}\left(T_{j}\right)$. It follows from (3.3), $y_{n}=\gamma_{n} u_{n}+\left(1-\gamma_{n}\right) W_{n} u_{n}$ and $u=W_{n} u$ such that

$$
\begin{aligned}
\left\|y_{n}-u\right\|^{2} & =\gamma_{n}\left\|u_{n}-u\right\|^{2}+\left(1-\gamma_{n}\right)\left\|W_{n} u_{n}-u\right\|^{2}-\gamma_{n}\left(1-\gamma_{n}\right)\left\|u_{n}-W_{n} u_{n}\right\|^{2} \\
& \leq \gamma_{n}\left\|u_{n}-u\right\|^{2}+\left(1-\gamma_{n}\right)\left[\left\|u_{n}-u\right\|^{2}+\varepsilon\left\|u_{n}-W_{n} u_{n}\right\|^{2}\right]-\gamma_{n}\left(1-\gamma_{n}\right)\left\|u_{n}-W_{n} u_{n}\right\|^{2} \\
& =\left\|u_{n}-u\right\|^{2}+\left(1-\gamma_{n}\right)\left(\varepsilon-\gamma_{n}\right)\left\|u_{n}-W_{n} u_{n}\right\|^{2} \\
& \leq\left\|u_{n}-u\right\|^{2}
\end{aligned}
$$

Put $M_{0}=\max \left\{\left\|x_{1}-u\right\|,(1 /(1-a))\|f(u)-u\|\right\}$. It is obvious that $\left\|x_{1}-u\right\| \leq M_{0}$. Suppose $\left\|x_{n}-u\right\| \leq M_{0}$. From (3.3), (3.5), and $x_{n+1}=\alpha_{n} f\left(x_{n}\right)+\beta_{n} x_{n}+\left(1-\alpha_{n}-\beta_{n}\right) y_{n}$, we have

$$
\begin{aligned}
\left\|x_{n+1}-u\right\| & =\left\|\alpha_{n} f\left(x_{n}\right)+\beta_{n} x_{n}+\left(1-\alpha_{n}-\beta_{n}\right) y_{n}-u\right\| \\
& \leq \alpha_{n}\left\|f\left(x_{n}\right)-f(u)\right\|+\alpha_{n}\|f(u)-u\|+\beta_{n}\left\|x_{n}-u\right\|+\left(1-\alpha_{n}-\beta_{n}\right)\left\|y_{n}-u\right\| \\
& \leq \alpha_{n} a\left\|x_{n}-u\right\|+\alpha_{n}\|f(u)-u\|+\beta_{n}\left\|x_{n}-u\right\|+\left(1-\alpha_{n}-\beta_{n}\right)\left\|u_{n}-u\right\| \\
& \leq \alpha_{n} a\left\|x_{n}-u\right\|+\alpha_{n}\|f(u)-u\|+\left(1-\alpha_{n}\right)\left\|x_{n}-u\right\| \\
& =(1-a) \alpha_{n} \frac{\|f(u)-u\|}{1-a}+\left[1-(1-a) \alpha_{n}\right]\left\|x_{n}-u\right\| \\
& \leq(1-a) \alpha_{n} M_{0}+\left[1-(1-a) \alpha_{n}\right] M_{0}=M_{0}
\end{aligned}
$$

for every $n=1,2, \ldots$. Therefore, $\left\{x_{n}\right\}$ is bounded. From (3.3) and (3.5), we also obtain that $\left\{y_{n}\right\}$ and $\left\{u_{n}\right\}$ are bounded.

Following [26], define $B_{n}: C \rightarrow C$ by

$$
B_{n}=\gamma_{n} I+\left(1-\gamma_{n}\right) W_{n}
$$


Fixed Point Theory and Applications

As shown in [26], each $B_{n}$ is a nonexpansive mapping on $C$. Set $M_{1}=\sup _{n \geq 1}\left\{\left\|u_{n}-W_{n} u_{n}\right\|\right\}$, we have

$$
\begin{aligned}
\left\|y_{n+1}-y_{n}\right\| & =\left\|B_{n+1}\left(u_{n+1}\right)-B_{n}\left(u_{n}\right)\right\| \\
& \leq\left\|B_{n+1}\left(u_{n+1}\right)-B_{n+1}\left(u_{n}\right)\right\|+\left\|B_{n+1}\left(u_{n}\right)-B_{n}\left(u_{n}\right)\right\| \\
& \leq\left\|u_{n+1}-u_{n}\right\|+M_{1}\left|\gamma_{n+1}-\gamma_{n}\right|+\left(1-\gamma_{n+1}\right)\left\|W_{n+1}\left(u_{n}\right)-W_{n}\left(u_{n}\right)\right\| \\
& \leq\left\|u_{n+1}-u_{n}\right\|+M_{1}\left|\gamma_{n+1}-\gamma_{n}\right|+\left(1-\gamma_{n+1}\right) \sum_{j=1}^{N}\left|\zeta_{j}^{(n+1)}-\zeta_{j}^{(n)}\right|\left\|T_{j} u_{n}\right\| .
\end{aligned}
$$

On the other hand, from $u_{n}=T_{r_{n}}\left(x_{n}\right)$ and $u_{n+1}=T_{r_{n+1}}\left(x_{n+1}\right)$, we have

$$
\begin{gathered}
F\left(u_{n}, y\right)+\varphi(y)+\frac{1}{r_{n}}\left\langle y-u_{n}, u_{n}-x_{n}\right\rangle \geq \varphi\left(u_{n}\right), \quad \forall y \in C, \\
F\left(u_{n+1}, y\right)+\varphi(y)+\frac{1}{r_{n+1}}\left\langle y-u_{n+1}, u_{n+1}-x_{n+1}\right\rangle \geq \varphi\left(u_{n+1}\right), \quad \forall y \in C .
\end{gathered}
$$

Putting $y=u_{n+1}$ in (3.9) and $y=u_{n}$ in (3.10), we have

$$
\begin{gathered}
F\left(u_{n}, u_{n+1}\right)+\varphi\left(u_{n+1}\right)+\frac{1}{r_{n}}\left\langle u_{n+1}-u_{n}, u_{n}-x_{n}\right\rangle \geq \varphi\left(u_{n}\right), \\
F\left(u_{n+1}, u_{n}\right)+\varphi\left(u_{n}\right)+\frac{1}{r_{n+1}}\left\langle u_{n}-u_{n+1}, u_{n+1}-x_{n+1}\right\rangle \geq \varphi\left(u_{n+1}\right) .
\end{gathered}
$$

So, from the monotonicity of $F$, we get

$$
\left\langle u_{n+1}-u_{n}, \frac{u_{n}-x_{n}}{r_{n}}-\frac{u_{n+1}-x_{n+1}}{r_{n+1}}\right\rangle \geq 0,
$$

hence

$$
\left\langle u_{n+1}-u_{n}, u_{n}-u_{n+1}+u_{n+1}-x_{n}-\frac{r_{n}}{r_{n+1}}\left(u_{n+1}-x_{n+1}\right)\right\rangle \geq 0
$$

Without loss of generality, let us assume that there exists a real number $b$ such that $r_{n}>b>0$ for all $n \in N$. Then,

$$
\begin{aligned}
\left\|u_{n+1}-u_{n}\right\|^{2} & \leq\left\langle u_{n+1}-u_{n}, x_{n+1}-x_{n}+\left(1-\frac{r_{n}}{r_{n+1}}\right)\left(u_{n+1}-x_{n+1}\right)\right\rangle \\
& \leq\left\|u_{n+1}-u_{n}\right\|\left\{\left\|x_{n+1}-x_{n}\right\|+\left|1-\frac{r_{n}}{r_{n+1}}\right|\left\|u_{n+1}-x_{n+1}\right\|\right\},
\end{aligned}
$$


hence

$$
\begin{aligned}
\left\|u_{n+1}-u_{n}\right\| & \leq\left\|x_{n+1}-x_{n}\right\|+\frac{1}{r_{n+1}}\left|r_{n+1}-r_{n}\right|\left\|u_{n+1}-x_{n+1}\right\| \\
& \leq\left\|x_{n+1}-x_{n}\right\|+\frac{1}{b}\left|r_{n+1}-r_{n}\right| M_{2}
\end{aligned}
$$

where $M_{2}=\sup \left\{\left\|u_{n}-x_{n}\right\|: n \geq 1\right\}$.

It follows from (3.8) and (3.15) that

$$
\begin{aligned}
\left\|y_{n+1}-y_{n}\right\| \leq & \left\|x_{n+1}-x_{n}\right\|+\frac{1}{b}\left|r_{n+1}-r_{n}\right| M_{2}+M_{1}\left|\gamma_{n+1}-\gamma_{n}\right| \\
& +\left(1-\gamma_{n+1}\right) \sum_{j=1}^{N}\left|\zeta_{j}^{(n+1)}-\zeta_{j}^{(n)}\right|\left\|T_{j} u_{n}\right\| .
\end{aligned}
$$

Define a sequence $\left\{v_{n}\right\}$ such that

$$
x_{n+1}=\beta_{n} x_{n}+\left(1-\beta_{n}\right) v_{n}, \quad \forall n \geq 1 .
$$

Then, we have

$$
\begin{aligned}
v_{n+1}-v_{n} & =\frac{x_{n+2}-\beta_{n+1} x_{n+1}}{1-\beta_{n+1}}-\frac{x_{n+1}-\beta_{n} x_{n}}{1-\beta_{n}} \\
& =\frac{\alpha_{n+1} f\left(x_{n+1}\right)+\left(1-\alpha_{n+1}-\beta_{n+1}\right) y_{n+1}}{1-\beta_{n+1}}-\frac{\alpha_{n} f\left(x_{n}\right)+\left(1-\alpha_{n}-\beta_{n}\right) y_{n}}{1-\beta_{n}} \\
& =\frac{\alpha_{n+1}}{1-\beta_{n+1}} f\left(x_{n+1}\right)-\frac{\alpha_{n}}{1-\beta_{n}} f\left(x_{n}\right)+y_{n+1}-y_{n}+\frac{\alpha_{n}}{1-\beta_{n}} y_{n}-\frac{\alpha_{n+1}}{1-\beta_{n+1}} y_{n+1} .
\end{aligned}
$$

From (3.18) and (3.16), we have

$$
\begin{aligned}
\| v_{n+1}- & v_{n}\|-\| x_{n+1}-x_{n} \| \\
\leq & \frac{\alpha_{n+1}}{1-\beta_{n+1}}\left(\left\|f\left(x_{n+1}\right)\right\|+\left\|y_{n+1}\right\|\right)+\frac{\alpha_{n}}{1-\beta_{n}}\left(\left\|f\left(x_{n}\right)\right\|+\left\|y_{n}\right\|\right) \\
& +\left\|y_{n+1}-y_{n}\right\|-\left\|x_{n+1}-x_{n}\right\| \\
\leq & \frac{\alpha_{n+1}}{1-\beta_{n+1}}\left(\left\|f\left(x_{n+1}\right)\right\|+\left\|y_{n+1}\right\|\right)+\frac{\alpha_{n}}{1-\beta_{n}}\left(\left\|f\left(x_{n}\right)\right\|+\left\|y_{n}\right\|\right) \\
& +\frac{1}{b}\left|r_{n+1}-r_{n}\right| M_{2}+M_{1}\left|\gamma_{n+1}-\gamma_{n}\right|+\left(1-\gamma_{n+1}\right) \sum_{j=1}^{N}\left|\zeta_{j}^{(n+1)}-\zeta_{j}^{(n)}\right|\left\|T_{j} u_{n}\right\| .
\end{aligned}
$$


Fixed Point Theory and Applications

It follows from $(\mathrm{C} 1)-(\mathrm{C} 5)$ that

$$
\limsup _{n \rightarrow \infty}\left(\left\|v_{n+1}-v_{n}\right\|-\left\|x_{n+1}-x_{n}\right\|\right) \leq 0
$$

Hence, by [27, Lemma 2.2], we have $\lim _{n \rightarrow \infty}\left\|v_{n}-x_{n}\right\|=0$. Consequently,

$$
\lim _{n \rightarrow \infty}\left\|x_{n+1}-x_{n}\right\|=\lim _{n \rightarrow \infty}\left(1-\beta_{n}\right)\left\|v_{n}-x_{n}\right\|=0 .
$$

Since $x_{n+1}=\alpha_{n} f\left(x_{n}\right)+\beta_{n} x_{n}+\left(1-\alpha_{n}-\beta_{n}\right) y_{n}$, we have

$$
\begin{aligned}
\left\|x_{n}-y_{n}\right\| & \leq\left\|x_{n+1}-x_{n}\right\|+\left\|x_{n+1}-y_{n}\right\| \\
& \leq\left\|x_{n+1}-x_{n}\right\|+\alpha_{n}\left\|f\left(x_{n}\right)-y_{n}\right\|+\beta_{n}\left\|x_{n}-y_{n}\right\|,
\end{aligned}
$$

thus

$$
\left\|x_{n}-y_{n}\right\| \leq \frac{1}{1-\beta_{n}}\left(\left\|x_{n+1}-x_{n}\right\|+\alpha_{n}\left\|f\left(x_{n}\right)-y_{n}\right\|\right) \text {. }
$$

It follows from (C1) and (C2) that $\lim _{n \rightarrow \infty}\left\|x_{n}-y_{n}\right\|=0$.

Since $x_{n+1}=\alpha_{n} f\left(x_{n}\right)+\beta_{n} x_{n}+\left(1-\alpha_{n}-\beta_{n}\right) y_{n}$, for $u \in \Omega$, it follows from (3.5) and (3.3) that

$$
\begin{aligned}
\left\|x_{n+1}-u\right\|^{2}= & \left\|\alpha_{n} f\left(x_{n}\right)+\beta_{n} x_{n}+\left(1-\alpha_{n}-\beta_{n}\right) y_{n}-u\right\|^{2} \\
\leq & \alpha_{n}\left\|f\left(x_{n}\right)-u\right\|^{2}+\beta_{n}\left\|x_{n}-u\right\|^{2}+\left(1-\alpha_{n}-\beta_{n}\right)\left\|y_{n}-u\right\|^{2} \\
\leq & \alpha_{n}\left\|f\left(x_{n}\right)-u\right\|^{2}+\beta_{n}\left\|x_{n}-u\right\|^{2} \\
& +\left(1-\alpha_{n}-\beta_{n}\right)\left[\left\|u_{n}-u\right\|^{2}+\left(1-\gamma_{n}\right)\left(\varepsilon-\gamma_{n}\right)\left\|u_{n}-W_{n} u_{n}\right\|^{2}\right] \\
\leq & \alpha_{n}\left\|f\left(x_{n}\right)-u\right\|^{2}+\left(1-\alpha_{n}\right)\left\|x_{n}-u\right\|^{2} \\
& +\left(1-\alpha_{n}-\beta_{n}\right)\left(1-\gamma_{n}\right)\left(\varepsilon-\gamma_{n}\right)\left\|u_{n}-W_{n} u_{n}\right\|^{2},
\end{aligned}
$$

from which it follows that

$$
\begin{aligned}
\left\|u_{n}-W_{n} u_{n}\right\|^{2} \leq & \frac{\alpha_{n}}{\left(1-\alpha_{n}-\beta_{n}\right)\left(1-\gamma_{n}\right)\left(\gamma_{n}-\varepsilon\right)}\left(\left\|f\left(x_{n}\right)-u\right\|^{2}-\left\|x_{n}-u\right\|^{2}\right) \\
& +\frac{1}{\left(1-\alpha_{n}-\beta_{n}\right)\left(1-\gamma_{n}\right)\left(\gamma_{n}-\varepsilon\right)}\left(\left\|x_{n}-u\right\|^{2}-\left\|x_{n+1}-u\right\|^{2}\right) . \\
\leq & \frac{\alpha_{n}}{\left(1-\alpha_{n}-\beta_{n}\right)\left(1-\gamma_{n}\right)\left(\gamma_{n}-\varepsilon\right)}\left(\left\|f\left(x_{n}\right)-u\right\|^{2}-\left\|x_{n}-u\right\|^{2}\right) \\
& +\frac{1}{\left(1-\alpha_{n}-\beta_{n}\right)\left(1-\gamma_{n}\right)\left(\gamma_{n}-\varepsilon\right)}\left(\left\|x_{n}-u\right\|+\left\|x_{n+1}-u\right\|\right)\left\|x_{n+1}-x_{n}\right\| .
\end{aligned}
$$


It follows from (C1)-(C3) and $\left\|x_{n+1}-x_{n}\right\| \rightarrow 0$ that

$$
\left\|u_{n}-W_{n} u_{n}\right\| \longrightarrow 0
$$

For $u \in \Omega$, we have from Lemma 2.2,

$$
\begin{aligned}
\left\|u_{n}-u\right\|^{2} & =\left\|S_{r_{n}} x_{n}-S_{r_{n}} u\right\|^{2} \leq\left\langle S_{r_{n}} x_{n}-S_{r_{n}} u, x_{n}-u\right\rangle \\
& =\left\langle u_{n}-u, x_{n}-u\right\rangle=\frac{1}{2}\left\{\left\|u_{n}-u\right\|^{2}+\left\|x_{n}-u\right\|^{2}-\left\|x_{n}-u_{n}\right\|^{2}\right\} .
\end{aligned}
$$

Hence,

$$
\left\|u_{n}-u\right\|^{2} \leq\left\|x_{n}-u\right\|^{2}-\left\|x_{n}-u_{n}\right\|^{2}
$$

By (3.24) and (3.28), we have

$$
\begin{aligned}
\left\|x_{n+1}-u\right\|^{2} & \leq \alpha_{n}\left\|f\left(x_{n}\right)-u\right\|^{2}+\beta_{n}\left\|x_{n}-u\right\|^{2}+\left(1-\alpha_{n}-\beta_{n}\right)\left\|u_{n}-u\right\|^{2} \\
& \leq \alpha_{n}\left\|f\left(x_{n}\right)-u\right\|^{2}+\beta_{n}\left\|x_{n}-u\right\|^{2}+\left(1-\alpha_{n}-\beta_{n}\right)\left[\left\|x_{n}-u\right\|^{2}-\left\|x_{n}-u_{n}\right\|^{2}\right] .
\end{aligned}
$$

Hence,

$$
\begin{aligned}
\left(1-\alpha_{n}-\beta_{n}\right)\left\|x_{n}-u_{n}\right\|^{2} \leq & \alpha_{n}\left\|f\left(x_{n}\right)-u\right\|^{2}-\alpha_{n}\left\|x_{n}-u\right\|^{2}+\left\|x_{n}-u\right\|^{2}-\left\|x_{n+1}-u\right\|^{2} \\
\leq & \alpha_{n}\left\|f\left(x_{n}\right)-u\right\|^{2}-\alpha_{n}\left\|x_{n}-u\right\|^{2} \\
& +\left(\left\|x_{n}-u\right\|+\left\|x_{n+1}-u\right\|\right)\left\|x_{n}-x_{n+1}\right\| .
\end{aligned}
$$

It follows from (C1), (C2), and $\left\|x_{n}-x_{n+1}\right\| \rightarrow 0$ that $\lim _{n \rightarrow \infty}\left\|x_{n}-u_{n}\right\|=0$.

Next, we show that

$$
\limsup _{n \rightarrow \infty}\left\langle f\left(u_{0}\right)-u_{0}, x_{n}-u_{0}\right\rangle \leq 0
$$

where $u_{0}=P_{\Omega} f\left(u_{0}\right)$. To show this inequality, we can choose a subsequence $\left\{x_{n_{i}}\right\}$ of $\left\{x_{n}\right\}$ such that

$$
\lim _{i \rightarrow \infty}\left\langle f\left(u_{0}\right)-u_{0}, x_{n_{i}}-u_{0}\right\rangle=\limsup _{n \rightarrow \infty}\left\langle f\left(u_{0}\right)-u_{0}, x_{n}-u_{0}\right\rangle .
$$

Since $\left\{x_{n_{i}}\right\}$ is bounded, there exists a subsequence $\left\{x_{n_{i_{i}}}\right\}$ of $\left\{x_{n_{i}}\right\}$ which converges weakly to $w$. Without loss of generality, we can assume that $\left\{x_{n_{i}}\right\} \rightarrow w$. From $\left\|x_{n}-u_{n}\right\| \rightarrow 0$, 
we obtain that $u_{n_{i}} \rightarrow w$. From $\left\|x_{n}-y_{n}\right\| \rightarrow 0$, we also obtain that $y_{n_{i}} \rightarrow w$. Since $\left\{u_{n_{i}}\right\} \subset C$ and $C$ is closed and convex, we obtain $w \in C$.

We first show that $w \in \bigcap_{k=1}^{N} \operatorname{Fix}\left(T_{k}\right)$. To see this, we observe that we may assume (by passing to a further subsequence if necessary) $\zeta_{k}^{\left(n_{i}\right)} \rightarrow \zeta_{k}$ (as $i \rightarrow \infty$ ) for $k=1,2, \ldots, N$. It is easy to see that $\zeta_{k}>0$ and $\sum_{k=1}^{N} \zeta_{k}=1$. We also have

$$
W_{n_{i}} x \longrightarrow W x \quad(\text { as } i \longrightarrow \infty) \forall x \in C,
$$

where $W=\sum_{k=1}^{N} \zeta_{k} T_{k}$. Note that by [21, Proposition 2.6], $W$ is an $\varepsilon$-strict pseudocontraction and $\operatorname{Fix}(W)=\bigcap_{i=1}^{N} \operatorname{Fix}\left(T_{i}\right)$. Since

$$
\begin{aligned}
\left\|u_{n_{i}}-W u_{n_{i}}\right\| & \leq\left\|u_{n_{i}}-W_{n_{i}} u_{n_{i}}\right\|+\left\|W_{n_{i}} u_{n_{i}}-W u_{n_{i}}\right\| \\
& \leq\left\|u_{n_{i}}-W_{n_{i}} u_{n_{i}}\right\|+\sum_{k=1}^{N}\left|\zeta_{k}^{\left(n_{i}\right)}-\zeta_{k}\right|\left\|T_{k} u_{n_{i}}\right\|,
\end{aligned}
$$

it follows from (3.26) and $\zeta_{k}^{\left(n_{i}\right)} \rightarrow \zeta_{k}$ that

$$
\left\|u_{n_{i}}-W u_{n_{i}}\right\| \longrightarrow 0
$$

So by the demiclosedness principle [21, Proposition 2.6(ii)], it follows that $w \in \operatorname{Fix}(W)=$ $\bigcap_{i=1}^{N} \operatorname{Fix}\left(T_{i}\right)$.

We now show $w \in \operatorname{GEP}(F, \varphi)$. By $u_{n}=T_{r_{n}} x_{n}$, we know that

$$
F\left(u_{n}, y\right)+\varphi(y)+\frac{1}{r_{n}}\left\langle y-u_{n}, u_{n}-x_{n}\right\rangle \geq \varphi\left(u_{n}\right), \quad \forall y \in C
$$

It follows from (A2) that

$$
\varphi(y)+\frac{1}{r_{n}}\left\langle y-u_{n}, u_{n}-x_{n}\right\rangle \geq F\left(y, u_{n}\right)+\varphi\left(u_{n}\right), \quad \forall y \in C
$$

Hence,

$$
\varphi(y)+\left\langle y-u_{n_{i}}, \frac{u_{n_{i}}-x_{n_{i}}}{r_{n_{i}}}\right\rangle \geq F\left(y, u_{n_{i}}\right)+\varphi\left(u_{n}\right), \quad \forall y \in C
$$


It follows from (A4), (A5) and the weakly lower semicontinuity of $\varphi,\left(u_{n_{i}}-x_{n_{i}}\right) / r_{n_{i}} \rightarrow$ 0 , and $u_{n_{i}} \rightarrow w$ that

$$
F(y, w)+\varphi(w) \leq \varphi(y), \quad \forall y \in C
$$

For $t$ with $0<t \leq 1$ and $y \in C \cap \operatorname{dom} \varphi$, let $y_{t}=t y+(1-t) w$. Since $y \in C \cap \operatorname{dom} \varphi$ and $w \in C \cap \operatorname{dom} \varphi$, we obtain $y_{t} \in C \cap \operatorname{dom} \varphi$, and hence $F\left(y_{t}, w\right)+\varphi(w) \leq \varphi\left(y_{t}\right)$. So by (A4) and the convexity of $\varphi$, we have

$$
\begin{aligned}
0 & =F\left(y_{t}, y_{t}\right)+\varphi\left(y_{t}\right)-\varphi\left(y_{t}\right) \\
& \leq t F\left(y_{t}, y\right)+(1-t) F\left(y_{t}, w\right)+t \varphi(y)+(1-t) \varphi(w)-\varphi\left(y_{t}\right) \\
& \leq t\left[F\left(y_{t}, y\right)+\varphi(y)-\varphi\left(y_{t}\right)\right] .
\end{aligned}
$$

Dividing by $t$, we get

$$
F\left(y_{t}, y\right)+\varphi(y)-\varphi\left(y_{t}\right) \geq 0
$$

Letting $t \rightarrow 0$, it follows from (A3) and the weakly lower semicontinuity of $\varphi$ that

$$
F(w, y)+\varphi(y) \geq \varphi(w)
$$

for all $y \in C \cap \operatorname{dom} \varphi$. Observe that if $y \in C \backslash \operatorname{dom} \varphi$, then $F(w, y)+\varphi(y) \geq \varphi(w)$ holds. Moreover, hence $w \in \operatorname{GEP}(F, \varphi)$. This implies $w \in \Omega$. Therefore, we have

$$
\limsup _{n \rightarrow \infty}\left\langle f\left(u_{0}\right)-u_{0}, x_{n}-u_{0}\right\rangle=\lim _{i \rightarrow \infty}\left\langle f\left(u_{0}\right)-u_{0}, x_{n_{i}}-u_{0}\right\rangle=\left\langle f\left(u_{0}\right)-u_{0}, w-u_{0}\right\rangle \leq 0 .
$$

Finally, we show that $x_{n} \rightarrow u_{0}$, where $u_{0}=P_{\Omega} f\left(u_{0}\right)$.

From Lemma 2.5, we have

$$
\begin{aligned}
\left\|x_{n+1}-u_{0}\right\|^{2}= & \left\|\alpha_{n}\left(f\left(x_{n}\right)-u_{0}\right)+\beta_{n}\left(x_{n}-u_{0}\right)+\left(1-\alpha_{n}-\beta_{n}\right)\left(y_{n}-u_{0}\right)\right\|^{2} \\
\leq & \left\|\beta_{n}\left(x_{n}-u_{0}\right)+\left(1-\alpha_{n}-\beta_{n}\right)\left(y_{n}-u_{0}\right)\right\|^{2}+2 \alpha_{n}\left\langle f\left(x_{n}\right)-u_{0}, x_{n+1}-u_{0}\right\rangle \\
\leq & {\left[\left(1-\alpha_{n}-\beta_{n}\right)\left\|y_{n}-u_{0}\right\|+\beta_{n}\left\|x_{n}-u_{0}\right\|\right]^{2}+2 \alpha_{n}\left\langle f\left(x_{n}\right)-u_{0}, x_{n+1}-u_{0}\right\rangle } \\
\leq & \left(1-\alpha_{n}\right)^{2}\left\|x_{n}-u_{0}\right\|^{2}+2 \alpha_{n}\left\langle f\left(x_{n}\right)-f\left(u_{0}\right), x_{n+1}-u_{0}\right\rangle \\
& +2 \alpha_{n}\left\langle f\left(u_{0}\right)-u_{0}, x_{n+1}-u_{0}\right\rangle \\
\leq & \left(1-\alpha_{n}\right)^{2}\left\|x_{n}-u_{0}\right\|^{2}+2 \alpha_{n} a\left\|x_{n}-u_{0}\right\|\left\|x_{n+1}-u_{0}\right\|+2 \alpha_{n}\left\langle f\left(u_{0}\right)-u_{0}, x_{n+1}-u_{0}\right\rangle \\
\leq & \left(1-\alpha_{n}\right)^{2}\left\|x_{n}-u_{0}\right\|^{2}+\alpha_{n} a\left(\left\|x_{n}-u_{0}\right\|^{2}+\left\|x_{n+1}-u_{0}\right\|^{2}\right) \\
& +2 \alpha_{n}\left\langle f\left(u_{0}\right)-u_{0}, x_{n+1}-u_{0}\right\rangle,
\end{aligned}
$$


thus

$$
\begin{aligned}
\left\|x_{n+1}-u_{0}\right\|^{2} \leq & \left(1-\frac{2(1-a) \alpha_{n}}{1-a \alpha_{n}}\right)\left\|x_{n}-u_{0}\right\|^{2} \\
& +\frac{2(1-a) \alpha_{n}}{1-a \alpha_{n}}\left\{\frac{\alpha_{n}}{2(1-a)}\left\|x_{n}-u_{0}\right\|^{2}+\frac{1}{1-a}\left\langle 2 f\left(u_{0}\right)-2 u_{0}, x_{n+1}-u_{0}\right\rangle\right\} .
\end{aligned}
$$

It follows from (C1), (3.43), (3.45), and Lemma 2.4 that $\lim _{n \rightarrow \infty}\left\|x_{n}-u_{0}\right\|=0$. From $\left\|x_{n}-u_{n}\right\| \rightarrow 0$ and $\left\|y_{n}-x_{n}\right\| \rightarrow 0$, we have $u_{n} \rightarrow u_{0}$ and $y_{n} \rightarrow u_{0}$. The proof is now complete.

Theorem 3.2. Let $C$ be a nonempty-closed convex subset of a real Hilbert space $H$. Let $F$ be a bifunction from $C \times C$ to $R$ satisfying (A1)-(A5), and let $\varphi: H \rightarrow R \cup\{+\infty\}$ be a proper lower semicontinuous and convex function such that $C \cap \operatorname{dom} \varphi \neq \varnothing$. Let $N \geq 1$ be an integer. For each $1 \leq j \leq N$, let $T_{j}: C \rightarrow C$ be an $\varepsilon_{j}$-strict pseudocontraction for some $0 \leq \varepsilon_{j}<1$ such that $\Omega=\bigcap_{j=1}^{N} \operatorname{Fix}\left(T_{j}\right) \cap \operatorname{GEP}(F, \varphi) \neq \varnothing$. Assume for each $n,\left\{\zeta_{j}^{(n)}\right\}_{j=1}^{N}$ is a finite sequence of positive numbers such that $\sum_{j=1}^{N} \zeta_{j}^{(n)}=1$ for all $n$ and $\inf _{n \geq 1} \zeta_{j}^{(n)}>0$ for all $0 \leq j \leq N$. Let $\varepsilon=\max \left\{\varepsilon_{j}: 1 \leq j \leq N\right\}$. Assume that either (B1) or (B2) holds. Let $v$ be an arbitrary point in $C$ and let $\left\{x_{n}\right\},\left\{u_{n}\right\}$, and $\left\{y_{n}\right\}$ be sequences generated by

$$
\begin{gathered}
x_{1}=x \in C \\
F\left(u_{n}, y\right)+\varphi(y)+\frac{1}{r_{n}}\left\langle y-u_{n}, u_{n}-x_{n}\right\rangle \geq \varphi\left(u_{n}\right), \quad \forall y \in C, \\
y_{n}=\gamma_{n} u_{n}+\left(1-\gamma_{n}\right) \sum_{j=1}^{N} \zeta_{j}^{(n)} T_{j} u_{n}, \\
x_{n+1}=\alpha_{n} v+\beta_{n} x_{n}+\left(1-\alpha_{n}-\beta_{n}\right) y_{n}
\end{gathered}
$$

for every $n=1,2, \ldots$, where $\left\{\gamma_{n}\right\},\left\{r_{n}\right\},\left\{\alpha_{n}\right\},\left\{\zeta_{1}^{(n)}\right\},\left\{\zeta_{2}^{(n)}\right\}, \ldots,\left\{\zeta_{N}^{(n)}\right\}$, and $\left\{\beta_{n}\right\}$ are sequences of numbers satisfying the conditions (C1)-(C5). Then, $\left\{x_{n}\right\},\left\{u_{n}\right\}$, and $\left\{y_{n}\right\}$ converge strongly to $w=$ $P_{\Omega} v$.

Proof. Let $f(x)=v$ for all $x \in C$, by Theorem 3.1, we obtain the desired result.

\section{Applications}

By Theorems 3.1 and 3.2, we can obtain many new and interesting strong convergence theorems. Now, give some examples as follows: for $j=1,2, \ldots, N$, let $T_{1}=T_{2}=\cdots=T_{N}=T$, by Theorems 3.1 and 3.2, respectively, we have the following results.

Theorem 4.1. Let $C$ be a nonempty-closed convex subset of a real Hilbert space $H$. Let $F$ be a bifunction from $C \times C$ to $R$ satisfying (A1)-(A5), and let $\varphi: H \rightarrow R \cup\{+\infty\}$ be a proper lower semicontinuous and convex function such that $C \cap \operatorname{dom} \varphi \neq \varnothing$. Let $T: C \rightarrow C$ be an $\varepsilon$-strict pseudocontraction for some $0 \leq \varepsilon<1$ such that $\operatorname{Fix}(T) \cap \operatorname{GEP}(F, \varphi) \neq \varnothing$. Assume that either (B1) or 
(B2) holds. Let $f$ be a contraction of $C$ into itself and let $\left\{x_{n}\right\},\left\{u_{n}\right\}$, and $\left\{y_{n}\right\}$ be sequences generated by

$$
\begin{gathered}
x_{1}=x \in C \\
F\left(u_{n}, y\right)+\varphi(y)+\frac{1}{r_{n}}\left\langle y-u_{n}, u_{n}-x_{n}\right\rangle \geq \varphi\left(u_{n}\right), \quad \forall y \in C \\
y_{n}=\gamma_{n} u_{n}+\left(1-\gamma_{n}\right) T u_{n} \\
x_{n+1}=\alpha_{n} f\left(x_{n}\right)+\beta_{n} x_{n}+\left(1-\alpha_{n}-\beta_{n}\right) y_{n}
\end{gathered}
$$

for every $n=1,2, \ldots$, where $\left\{\gamma_{n}\right\},\left\{r_{n}\right\},\left\{\alpha_{n}\right\}$, and $\left\{\beta_{n}\right\}$ are sequences of numbers satisfying the conditions (C1)-(C4). Then, $\left\{x_{n}\right\},\left\{u_{n}\right\}$, and $\left\{y_{n}\right\}$ converge strongly to $w=P_{\operatorname{Fix}(T) \cap \operatorname{GEP}(F, \varphi)} f(w)$.

Theorem 4.2. Let $C$ be a nonempty-closed convex subset of a real Hilbert space $H$. Let $F$ be a bifunction from $C \times C$ to $R$ satisfying (A1)-(A5), and let $\varphi: H \rightarrow R \cup\{+\infty\}$ be a proper lower semicontinuous and convex function such that $C \cap \operatorname{dom} \varphi \neq \varnothing$. Let $T: C \rightarrow C$ be an $\varepsilon$-strict pseudocontraction for some $0 \leq \varepsilon<1$ such that $\operatorname{Fix}(T) \cap \operatorname{GEP}(F, \varphi) \neq \varnothing$. Assume that either (B1) or (B2) holds. Let $v$ be an arbitrary point in $C$, and let $\left\{x_{n}\right\},\left\{u_{n}\right\}$, and $\left\{y_{n}\right\}$ be sequences generated by

$$
\begin{gathered}
x_{1}=x \in C \\
F\left(u_{n}, y\right)+\varphi(y)+\frac{1}{r_{n}}\left\langle y-u_{n}, u_{n}-x_{n}\right\rangle \geq \varphi\left(u_{n}\right), \quad \forall y \in C \\
y_{n}=\gamma_{n} u_{n}+\left(1-\gamma_{n}\right) T u_{n} \\
x_{n+1}=\alpha_{n} v+\beta_{n} x_{n}+\left(1-\alpha_{n}-\beta_{n}\right) y_{n}
\end{gathered}
$$

for every $n=1,2, \ldots$, where $\left\{\gamma_{n}\right\},\left\{r_{n}\right\},\left\{\alpha_{n}\right\}$, and $\left\{\beta_{n}\right\}$ are sequences of numbers satisfying the conditions $(C 1)-(C 4)$. Then, $\left\{x_{n}\right\},\left\{u_{n}\right\}$, and $\left\{y_{n}\right\}$ converge strongly to $w=P_{\operatorname{Fix}(T) \cap \operatorname{GEP}(F, \varphi)} v$.

We need the following two assumptions.

(B3) For each $x \in H$ and $r>0$, there exist a bounded subset $D_{x} \subseteq C$ and $y_{x} \in C$ such that for any $z \in C \backslash D_{x}$,

$$
F\left(z, y_{x}\right)+\frac{1}{r}\left\langle y_{x}-z, z-x\right\rangle<0
$$

(B4) For each $x \in H$ and $r>0$, there exist a bounded subset $D_{x} \subseteq C$ and $y_{x} \in C \cap \operatorname{dom} \varphi$ such that for any $z \in C \backslash D_{x}$,

$$
g\left(y_{x}\right)+\varphi\left(y_{x}\right)+\frac{1}{r}\left\langle y_{x}-z, z-x\right\rangle<\varphi(z)+g(z)
$$

Let $\varphi(x)=\delta_{C}(x), \forall x \in H$, by Theorems 3.1 and 3.2, respectively, we obtain the following results. 
Theorem 4.3. Let $C$ be a nonempty-closed convex subset of a real Hilbert space $H$. Let $F$ be a bifunction from $C \times C$ to $R$ satisfying (A1)-(A5). Let $N \geq 1$ be an integer. For each $1 \leq j \leq N$, let $T_{j}$ : $C \rightarrow C$ be an $\varepsilon_{j}$-strict pseudocontraction for some $0 \leq \varepsilon_{j}<1$ such that $\Gamma=\bigcap_{j=1}^{N} \operatorname{Fix}\left(T_{j}\right) \cap E P(F) \neq \varnothing$. Assume for each $n,\left\{\zeta_{j}^{(n)}\right\}_{j=1}^{N}$ is a finite sequence of positive numbers such that $\sum_{j=1}^{N} \zeta_{j}^{(n)}=1$ for all $n$ and $\inf _{n \geq 1} \zeta_{j}^{(n)}>0$ for all $0 \leq j \leq N$. Let $\varepsilon=\max \left\{\varepsilon_{j}: 1 \leq j \leq N\right\}$. Assume that either (B3) or (B2) holds. Let $f$ be a contraction of $H$ into itself, and let $\left\{x_{n}\right\},\left\{u_{n}\right\}$, and $\left\{y_{n}\right\}$ be sequences generated by

$$
\begin{gathered}
x_{1}=x \in C, \\
F\left(u_{n}, y\right)+\frac{1}{r_{n}}\left\langle y-u_{n}, u_{n}-x_{n}\right\rangle \geq 0, \quad \forall y \in C, \\
y_{n}=\gamma_{n} u_{n}+\left(1-\gamma_{n}\right) \sum_{j=1}^{N} \zeta_{j}^{(n)} T_{j} u_{n}, \\
x_{n+1}=\alpha_{n} f\left(x_{n}\right)+\beta_{n} x_{n}+\left(1-\alpha_{n}-\beta_{n}\right) y_{n}
\end{gathered}
$$

for every $n=1,2, \ldots$, where $\left\{\gamma_{n}\right\},\left\{r_{n}\right\},\left\{\alpha_{n}\right\},\left\{\zeta_{1}^{(n)}\right\},\left\{\zeta_{2}^{(n)}\right\}, \ldots,\left\{\zeta_{N}^{(n)}\right\}$, and $\left\{\beta_{n}\right\}$ are sequences of numbers satisfying the conditions (C1)-(C5). Then, $\left\{x_{n}\right\},\left\{u_{n}\right\}$, and $\left\{y_{n}\right\}$ converge strongly to $w=$ $P_{\Gamma} f(w)$.

Theorem 4.4. Let $C$ be a nonempty-closed convex subset of a real Hilbert space $H$. Let $F$ be a bifunction from $C \times C$ to $R$ satisfying (A1)-(A5). Let $N \geq 1$ be an integer. For each $1 \leq j \leq N$, let $T_{j}$ : $C \rightarrow C$ be an $\varepsilon_{j}$-strict pseudocontraction for some $0 \leq \varepsilon_{j}<1$ such that $\Gamma=\bigcap_{j=1}^{N} \operatorname{Fix}\left(T_{j}\right) \cap E P(F) \neq \varnothing$. Assume for each $n,\left\{\zeta_{j}^{(n)}\right\}_{j=1}^{N}$ is a finite sequence of positive numbers such that $\sum_{j=1}^{N} \zeta_{j}^{(n)}=1$ for all $n$ and $\inf _{n \geq 1} \zeta_{j}^{(n)}>0$ for all $0 \leq j \leq N$. Let $\varepsilon=\max \left\{\varepsilon_{j}: 1 \leq j \leq N\right\}$. Assume that either (B3) or (B2) holds. Let $v$ be an arbitrary point in $C$, and let $\left\{x_{n}\right\},\left\{u_{n}\right\}$, and $\left\{y_{n}\right\}$ be sequences generated by

$$
\begin{gathered}
x_{1}=x \in C, \\
F\left(u_{n}, y\right)+\frac{1}{r_{n}}\left\langle y-u_{n}, u_{n}-x_{n}\right\rangle \geq 0, \quad \forall y \in C, \\
y_{n}=\gamma_{n} u_{n}+\left(1-\gamma_{n}\right) \sum_{j=1}^{N} \zeta_{j}^{(n)} T_{j} u_{n}, \\
x_{n+1}=\alpha_{n} v+\beta_{n} x_{n}+\left(1-\alpha_{n}-\beta_{n}\right) y_{n}
\end{gathered}
$$

for every $n=1,2, \ldots$, where $\left\{\gamma_{n}\right\},\left\{r_{n}\right\},\left\{\alpha_{n}\right\},\left\{\zeta_{1}^{(n)}\right\},\left\{\zeta_{2}^{(n)}\right\}, \ldots,\left\{\zeta_{N}^{(n)}\right\}$, and $\left\{\beta_{n}\right\}$ are sequences of numbers satisfying the conditions (C1)-(C5). Then, $\left\{x_{n}\right\},\left\{u_{n}\right\}$, and $\left\{y_{n}\right\}$ converge strongly to $w=$ $P_{\Gamma} v$.

Let $F(x, y)=g(y)-g(x)$ for all $x, y \in C$, by Theorems 3.1 and 3.2, respectively, we obtain the following results.

Theorem 4.5. Let $C$ be a nonempty-closed convex subset of a real Hilbert space $H$. Let $g: C \rightarrow R$ be a lower semicontinuous and convex function, and let $\varphi: H \rightarrow R \cup\{+\infty\}$ be a proper lower semicontinuous and convex function such that $C \cap \operatorname{dom} \varphi \neq \varnothing$. Let $N \geq 1$ be an integer. For each 
$1 \leq j \leq N$, let $T_{j}: C \rightarrow C$ be an $\varepsilon_{j}$-strict pseudocontraction for some $0 \leq \varepsilon_{j}<1$ such that $\Theta=$ $\bigcap_{j=1}^{N} \operatorname{Fix}\left(T_{j}\right) \cap \operatorname{Argmin}(g, \varphi) \neq \varnothing$. Assume for each $n,\left\{\zeta_{j}^{(n)}\right\}_{j=1}^{N}$ is a finite sequence of positive numbers such that $\sum_{j=1}^{N} \zeta_{j}^{(n)}=1$ for all $n$ and $\inf _{n \geq 1} \zeta_{j}^{(n)}>0$ for all $0 \leq j \leq N$. Let $\varepsilon=\max \left\{\varepsilon_{j}: 1 \leq j \leq N\right\}$. Assume that either (B4) or (B2) holds. Let $f$ be a contraction of $H$ into itself, and let $\left\{x_{n}\right\},\left\{u_{n}\right\}$ and $\left\{y_{n}\right\}$ be sequences generated by

$$
\begin{gathered}
x_{1}=x \in C, \\
g(y)+\varphi(y)+\frac{1}{r_{n}}\left\langle y-u_{n}, u_{n}-x_{n}\right\rangle \geq g\left(u_{n}\right)+\varphi\left(u_{n}\right), \quad \forall y \in C, \\
y_{n}=\gamma_{n} u_{n}+\left(1-\gamma_{n}\right) \sum_{j=1}^{N} \zeta_{j}^{(n)} T_{j} u_{n} \\
x_{n+1}=\alpha_{n} f\left(x_{n}\right)+\beta_{n} x_{n}+\left(1-\alpha_{n}-\beta_{n}\right) y_{n}
\end{gathered}
$$

for every $n=1,2, \ldots$, where $\left\{\gamma_{n}\right\},\left\{r_{n}\right\},\left\{\alpha_{n}\right\},\left\{\zeta_{1}^{(n)}\right\},\left\{\zeta_{2}^{(n)}\right\}, \ldots,\left\{\zeta_{N}^{(n)}\right\}$, and $\left\{\beta_{n}\right\}$ are sequences of numbers satisfying the conditions (C1)-(C5). Then, $\left\{x_{n}\right\},\left\{u_{n}\right\}$, and $\left\{y_{n}\right\}$ converge strongly to $w=$ $P_{\Theta} f(w)$.

Theorem 4.6. Let $C$ be a nonempty-closed convex subset of a real Hilbert space $H$. Let $g: C \rightarrow R$ be a lower semicontinuous and convex function, and let $\varphi: H \rightarrow R \cup\{+\infty\}$ be a proper lower semicontinuous and convex function such that $C \cap \operatorname{dom} \varphi \neq \varnothing$. Let $N \geq 1$ be an integer. For each $1 \leq j \leq N$, let $T_{j}: C \rightarrow C$ be an $\varepsilon_{j}$-strict pseudocontraction for some $0 \leq \varepsilon_{j}<1$ such that $\Theta=$ $\bigcap_{j=1}^{N} \operatorname{Fix}\left(T_{j}\right) \cap \operatorname{Argmin}(g, \varphi) \neq \varnothing$. Assume for each $n,\left\{\zeta_{j}^{(n)}\right\}_{j=1}^{N}$ is a finite sequence of positive numbers such that $\sum_{j=1}^{N} \zeta_{j}^{(n)}=1$ for all $n$ and $\inf _{n \geq 1} \zeta_{j}^{(n)}>0$ for all $0 \leq j \leq N$. Let $\varepsilon=\max \left\{\varepsilon_{j}: 1 \leq j \leq N\right\}$. Assume that either (B4) or (B2) holds. Let $v$ be an arbitrary point in $C$, and let $\left\{x_{n}\right\},\left\{u_{n}\right\}$, and $\left\{y_{n}\right\}$ be sequences generated by

$$
\begin{gathered}
x_{1}=x \in C \\
g(y)+\varphi(y)+\frac{1}{r_{n}}\left\langle y-u_{n}, u_{n}-x_{n}\right\rangle \geq \varphi\left(u_{n}\right)+g\left(u_{n}\right), \quad \forall y \in C, \\
y_{n}=\gamma_{n} u_{n}+\left(1-\gamma_{n}\right) \sum_{j=1}^{N} \zeta_{j}^{(n)} T_{j} u_{n} \\
x_{n+1}=\alpha_{n} v+\beta_{n} x_{n}+\left(1-\alpha_{n}-\beta_{n}\right) y_{n}
\end{gathered}
$$

for every $n=1,2, \ldots$, where $\left\{\gamma_{n}\right\},\left\{r_{n}\right\},\left\{\alpha_{n}\right\},\left\{\zeta_{1}^{(n)}\right\},\left\{\zeta_{2}^{(n)}\right\}, \ldots,\left\{\zeta_{N}^{(n)}\right\}$, and $\left\{\beta_{n}\right\}$ are sequences of numbers satisfying the conditions (C1)-(C5). Then, $\left\{x_{n}\right\},\left\{u_{n}\right\}$, and $\left\{y_{n}\right\}$ converge strongly to $w=$ $P_{\Theta} v$.

Let $\varphi(x)=\delta_{C}(x), \forall x \in H$, and let $F(x, y)=0$ for all $x, y \in C$. Then $u_{n}=P_{C} x_{n}=x_{n}$. By Theorems 3.1 and 3.2, we obtain the following results.

Theorem 4.7. Let $C$ be a nonempty-closed convex subset of a real Hilbert space $H$. Let $N \geq 1$ be an integer. For each $1 \leq j \leq N$, let $T_{j}: C \rightarrow C$ be an $\varepsilon_{j}$-strict pseudocontraction for some $0 \leq \varepsilon_{j}<1$ 
such that $\bigcap_{j=1}^{N} \operatorname{Fix}\left(T_{j}\right) \neq \varnothing$. Assume for each $n,\left\{\zeta_{j}^{(n)}\right\}_{j=1}^{N}$ is a finite sequence of positive numbers such that $\sum_{j=1}^{N} \zeta_{j}^{(n)}=1$ for all $n$ and $\inf _{n \geq 1} \zeta_{j}^{(n)}>0$ for all $0 \leq j \leq N$. Let $\varepsilon=\max \left\{\varepsilon_{j}: 1 \leq j \leq N\right\}$. Let $f$ be a contraction of $H$ into itself, and let $\left\{x_{n}\right\}$ and $\left\{y_{n}\right\}$ be sequences generated by

$$
\begin{gathered}
x_{1}=x \in C, \\
y_{n}=\gamma_{n} x_{n}+\left(1-\gamma_{n}\right) \sum_{j=1}^{N} \zeta_{j}^{(n)} T_{j} x_{n}, \\
x_{n+1}=\alpha_{n} f\left(x_{n}\right)+\beta_{n} x_{n}+\left(1-\alpha_{n}-\beta_{n}\right) y_{n}
\end{gathered}
$$

for every $n=1,2, \ldots$, where $\left\{\gamma_{n}\right\},\left\{\alpha_{n}\right\},\left\{\zeta_{1}^{(n)}\right\},\left\{\zeta_{2}^{(n)}\right\}, \ldots,\left\{\zeta_{N}^{(n)}\right\}$, and $\left\{\beta_{n}\right\}$ are sequences of numbers satisfying the conditions (C1)-(C3) and (C5). Then, $\left\{x_{n}\right\}$, and $\left\{y_{n}\right\}$ converge strongly to $w=$ $P_{\cap_{j=1}^{N} \operatorname{Fix}\left(T_{j}\right)} f(w)$.

Theorem 4.8. Let $C$ be a nonempty-closed convex subset of a real Hilbert space $H$. Let $N \geq 1$ be an integer. For each $1 \leq j \leq N$, let $T_{j}: C \rightarrow C$ be an $\varepsilon_{j}$-strict pseudocontraction for some $0 \leq \varepsilon_{j}<1$ such that $\bigcap_{j=1}^{N} \operatorname{Fix}\left(T_{j}\right) \neq \varnothing$. Assume for each $n,\left\{\zeta_{j}^{(n)}\right\}_{j=1}^{N}$ is a finite sequence of positive numbers such that $\sum_{j=1}^{N} \zeta_{j}^{(n)}=1$ for all $n$ and $\inf _{n \geq 1} \zeta_{j}^{(n)}>0$ for all $0 \leq j \leq N$. Let $\varepsilon=\max \left\{\varepsilon_{j}: 1 \leq j \leq N\right\}$. Let $v$ be an arbitrary point in $C$, and let $\left\{x_{n}\right\}$ and $\left\{y_{n}\right\}$ be sequences generated by

$$
\begin{gathered}
x_{1}=x \in C, \\
y_{n}=\gamma_{n} x_{n}+\left(1-\gamma_{n}\right) \sum_{j=1}^{N} \zeta_{j}^{(n)} T_{j} x_{n}, \\
x_{n+1}=\alpha_{n} v+\beta_{n} x_{n}+\left(1-\alpha_{n}-\beta_{n}\right) y_{n}
\end{gathered}
$$

for every $n=1,2, \ldots$, where $\left\{\gamma_{n}\right\},\left\{\alpha_{n}\right\},\left\{\zeta_{1}^{(n)}\right\},\left\{\zeta_{2}^{(n)}\right\}, \ldots,\left\{\zeta_{N}^{(n)}\right\}$, and $\left\{\beta_{n}\right\}$ are sequences of numbers satisfying the conditions (C1)-(C3) and (C5). Then, $\left\{x_{n}\right\}$ and $\left\{y_{n}\right\}$ converge strongly to $w=$ $P_{\cap_{j=1}^{N} \operatorname{Fix}\left(T_{j}\right)} v$.

Remark 4.9. (1) Since the nonexpansive mappings have been replaced by the strict pseudocontractions, Theorems 3.1, 3.2, 4.1 and 4.2 extend and improve [6, Theorem 3.1], [8, Theorem 3.5], [9, Theorems 4.1 and 4.2], [18, Theorem 4.1], and the main results in [9-11, 13$16]$.

(2) Since the weak convergence has been replaced by strong convergence, Theorems 3.1, 3.2, 4.1-4.4 extend and improve [12, Theorem 3.1], [10, Corollary 4.1].

(3) Theorems 4.7 and 4.8 are strong convergence theorems for strict pseudocontractions without $C Q$ constraints and hence they improve the corresponding results in $[19,21]$. Theorems 3.1 and 3.2 also improve [10, Corollary 3.1].

\section{Acknowledgments}

The first author was supported by the National Natural Science Foundation of China (Grants No. 10771228 and No. 10831009), the Science and Technology Research Project of 
Chinese Ministry of Education (Grant no. 206123), the Education Committee project Research Foundation of Chongqing Normal University (Grant no. KJ070816); the second and third authors were partially supported by the Grants NSC97-2221-E-230-017 and NSC96-2628-E110-014-MY3, respectively.

\section{References}

[1] F. Flores-Bazán, “Existence theorems for generalized noncoercive equilibrium problems: the quasiconvex case," SIAM Journal on Optimization, vol. 11, no. 3, pp. 675-690, 2000.

[2] G. Bigi, M. Castellani, and G. Kassay, "A dual view of equilibrium problems," Journal of Mathematical Analysis and Applications, vol. 342, no. 1, pp. 17-26, 2008.

[3] A. N. Iusem and W. Sosa, "Iterative algorithms for equilibrium problems," Optimization, vol. 52, no. 3, pp. 301-316, 2003.

[4] S. D. Flåm and A. S. Antipin, "Equilibrium programming using proximal-like algorithms," Mathematical Programming, vol. 78, no. 1, pp. 29-41, 1997.

[5] E. Blum and W. Oettli, "From optimization and variational inequalities to equilibrium problems," The Mathematics Student, vol. 63, no. 1-4, pp. 123-145, 1994.

[6] L.-C. Ceng and J.-C. Yao, "A hybrid iterative scheme for mixed equilibrium problems and fixed point problems," Journal of Computational and Applied Mathematics, vol. 214, no. 1, pp. 186-201, 2008.

[7] F. E. Browder and W. V. Petryshyn, "Construction of fixed points of nonlinear mappings in Hilbert space," Journal of Mathematical Analysis and Applications, vol. 20, pp. 197-228, 1967.

[8] Y. Yao, Y.-C. Liou, and J.-C. Yao, "A new hybrid iterative algorithm for fixed-point problems, variational inequality problems, and mixed equilibrium problems," Fixed Point Theory and Applications, vol. 2008, Article ID 417089, 15 pages, 2008.

[9] J.-W. Peng and J.-C. Yao, "A new hybrid-extragradient method for generalized mixed equilibrium problems, fixed point problems and variational inequality problems," Taiwanese Journal of Mathematics, vol. 12, no. 6, pp. 1401-1432, 2008.

[10] J.-W. Peng and J.-C. Yao, "Some new iterative algorithms for generalized mixed equilibrium problems with strict pseudo-contractions and monotone mappings," to appear in Taiwanese Journal of Mathematics.

[11] P. L. Combettes and S. A. Hirstoaga, "Equilibrium programming in Hilbert spaces," Journal of Nonlinear and Convex Analysis, vol. 6, no. 1, pp. 117-136, 2005.

[12] S. Takahashi and W. Takahashi, "Viscosity approximation methods for equilibrium problems and fixed point problems in Hilbert spaces," Journal of Mathematical Analysis and Applications, vol. 331, no. 1, pp. 506-515, 2007.

[13] Y. Su, M. Shang, and X. Qin, "An iterative method of solution for equilibrium and optimization problems," Nonlinear Analysis: Theory, Methods E Applications, vol. 69, no. 8, pp. 2709-2719, 2008.

[14] A. Tada and W. Takahashi, "Weak and strong convergence theorems for a nonexpansive mapping and an equilibrium problem," Journal of Optimization Theory and Applications, vol. 133, no. 3, pp. 359-370, 2007.

[15] L.-C. Ceng, S. AI-Homidan, Q. H. Ansari, and J.-C. Yao, "An iterative scheme for equilibrium problems and fixed point problems of strict pseudo-contraction mappings," Journal of Computational and Applied Mathematics, vol. 223, no. 2, pp. 967-974, 2009.

[16] S. S. Chang, H. W. Joseph Lee, and C. K. Chan, "A new method for solving equilibrium problem, fixed point problem and variational inequality problem with application to optimization," Nonlinear Analysis: Theory, Methods E Applications. In press.

[17] V. Colao, G. Marino, and H.-K. Xu, "An iterative method for finding common solutions of equilibrium and fixed point problems," Journal of Mathematical Analysis and Applications, vol. 344, no. 1, pp. 340$352,2008$.

[18] S. Takahashi and W. Takahashi, "Strong convergence theorem for a generalized equilibrium problem and a nonexpansive mapping in a Hilbert space," Nonlinear Analysis: Theory, Methods E Applications, vol. 69, no. 3, pp. 1025-1033, 2008.

[19] G. Marino and H.-K. Xu, "Weak and strong convergence theorems for strict pseudo-contractions in Hilbert spaces," Journal of Mathematical Analysis and Applications, vol. 329, no. 1, pp. 336-346, 2007.

[20] H. Zhou, "Convergence theorems of fixed points for $\kappa$-strict pseudo-contractions in Hilbert spaces," Nonlinear Analysis: Theory, Methods E Applications, vol. 69, no. 2, pp. 456-462, 2008. 
[21] G. L. Acedo and H.-K. Xu, "Iterative methods for strict pseudo-contractions in Hilbert spaces," Nonlinear Analysis: Theory, Methods E Applications, vol. 67, no. 7, pp. 2258-2271, 2007.

[22] K. Fan, "A generalization of Tychonoff's fixed point theorem," Mathematische Annalen, vol. 142, pp. 305-310, 1961.

[23] K. Goebel and S. Reich, Uniform Convexity, Hyperbolic Geometry, and Nonexpansive Mappings, vol. 83 of Monographs and Textbooks in Pure and Applied Mathematics, Marcel Dekker, New York, NY, USA, 1984.

[24] H.-K. Xu, "Iterative algorithms for nonlinear operators," Journal of the London Mathematical Society. Second Series, vol. 66, no. 1, pp. 240-256, 2002.

[25] H. K. Xu, "An iterative approach to quadratic optimization," Journal of Optimization Theory and Applications, vol. 116, no. 3, pp. 659-678, 2003.

[26] G. Marino, V. Colao, X. Qin, and S. M. Kang, "Strong convergence of the modified Mann iterative method for strict pseudo-contractions," Computers \& Mathematics with Applications, vol. 57, no. 3, pp. 455-465, 2009.

[27] T. Suzuki, "Strong convergence theorems for infinite families of nonexpansive mappings in general Banach spaces," Fixed Point Theory and Applications, vol. 2005, no. 1, pp. 103-123, 2005. 